Schneider, Uwe; Frank, Birgit; Fiedler, Anja; Kaehler, Christiane; Hoyer, Dirk; Liehr, Mario; Haueisen, Jens; Schleussner, Ekkehard:

\title{
Human fetal heart rate variability-characteristics of autonomic regulation in the third trimester of gestation
}

URN: $\quad$ urn:nbn:de:gbv:ilm1-2015210182

Published OpenAccess: January 2015

Original published in:

Journal of perinatal medicine : official journal of the World Association of Perinatal Medicine. - Berlin [u.a.] : de Gruyter (ISSN 1619-3997). - 36 (2008) 5, S. 433-441.

DOI: $\quad$ 10.1515/JPM.2008.059

URL: $\quad$ http://dx.doi.org/10.1515/JPM.2008.059

[Visited: 2014-10-15]

„Im Rahmen der hochschulweiten Open-Access-Strategie für die Zweitveröffentlichung identifiziert durch die Universitätsbibliothek Ilmenau."

"Within the academic Open Access Strategy identified for deposition by Ilmenau University Library."

"Dieser Beitrag ist mit Zustimmung des Rechteinhabers aufgrund einer (DFG-geförderten) Allianz- bzw. Nationallizenz frei zugänglich."

"This publication is with permission of the rights owner freely accessible due to an Alliance licence and a national licence (funded by the DFG, German Research Foundation) respectively."

\section{DFG}

Nationallizenzen 


\section{Human fetal heart rate variability-characteristics of autonomic regulation in the third trimester of gestation ${ }^{\star, \star \star}$}

\author{
Uwe Schneider ${ }^{1, \star \star *}$, Birgit Frank ${ }^{2}$, Anja Fiedler ${ }^{1}$, \\ Christiane Kaehler ${ }^{3}$, Dirk Hoyer', Mario Liehr², \\ Jens Haueisen ${ }^{2,4}$ and Ekkehard Schleussner ${ }^{1}$ \\ ${ }^{1}$ University Hospital, Department of Obstetrics, \\ Friedrich Schiller University of Jena, Germany \\ 2 University Hospital, Biomagnetic Center, Department \\ of Neurology, Friedrich Schiller University of Jena, \\ Germany \\ ${ }^{3}$ Center for Ultrasound and Prenatal Diagnosis, Erfurt, \\ Germany \\ ${ }^{4}$ Institute of Biomedical Engineering and Informatics, \\ Technical University of IImenau, Germany
}

complexity measure. SDNN/RMSSD was introduced as a potential marker for sympatho-vagal balance.

Results: Low activity fHRP (I) were characterized by significantly lower level fHRV indices and higher PE_Mean when compared to fHRP II. We found that SDNN/RMSSD decreases with gestation in fHRP I, which suggests increasing vagal influence. In fHRP III (assigned to active awake fetuses only after 32 weeks), highest level SDNN and $\mathrm{mHR}$ are associated with a dramatically reduced complexity. fHRV indices cluster characteristically with the activity levels.

Conclusions: We conclude that a combined analysis of fHRV, based on SDNN/RMSSD and PE_Mean, and fHRP is advantageous in the assessment of maturation of the fetal autonomic nervous system.

Keywords: Autonomic nervous system; fetal magnetocardiography; fetal state; heart rate variability; sympathovagal balance.

\section{Introduction}

The fetal heart rate (fHR) is the primary variable of controlling the cardiac stability of the yet unborn fetus, and is the functional parameter easiest for non-invasive access. The fHR is under control of the developing autonomic nervous system (ANS). FHR monitoring enables continued verification of the health, vitality, and fetal wellbeing [10]. Furthermore, fHR monitoring also provides important information on the developmental stages of the fetal ANS.

The vast majority of data on the development of fHR regulation has been derived from Doppler ultrasoundbased cardiotocography systems (CTG). In comparison to the CTG, electrophysiological methods such as fetal electrocardiography or fetal magnetocardiography (fMCG) are able to enhance the temporal acuity of fHR monitoring [14]. The temporal resolution of the QRS complex detection enables precise fetal beat-to-beat heart rate variability (fHRV) analysis. Considering the upper boundary of the vagal modulated fetal high frequency band (HF: 0.4-1.7 Hz) [3], CTG systems are not able to optimally analyze the faster autonomic rhythms. Hence, fHRV analysis based on an fMCG temporal solution is likely to enhance our knowledge on the development of the fetal ANS.

Interpretation of the fHR must take into account changes in the fHR patterns with increasing gestational 
age (GA) [15], but the same fetus shows variant patterns of fHR depending on if it is in an active or quiet state [16]. These rest/activity cycles have been described from 23 weeks of GA onwards [15]. The progressive synchronization of variables of the fetal state (fHR pattern, body movements, eye movements) are markers of the developmental integrity of the fetus [4]. Determination of the state of the fetal development is based on the evaluation of conventional CTG recordings accompanied by continuous B-mode ultrasound observations [4, 15, 16]. Four states have been postulated: "quiet sleep" (1F), "active sleep" (2F), "quiet wakefulness" (3F) and "active waking" (4F). Criteria which classify the typical heart rate patterns (fHRP) that characterize these states have been primarily applied from 32 completed weeks of GA onwards [11]. Beyond 35 completed weeks of GA the state variables characteristically coincide for the states $1 \mathrm{~F}, 2 \mathrm{~F}$ and $4 \mathrm{~F}$ at $85-90 \%$ of the time share $[5,11,16]$. The $3 F$ state, though, was observed so rarely that its existence has been doubted or neglected [4, 16, 17]. Consequently, fHRP strongly correlate to the varying states of fetal activity in the third trimester of gestation and the lack of other variables does not invalidate the new information obtained by high resolution fHR analysis.

$\mathrm{HRV}$ analysis is an internationally standardized procedure to assess the vegetative regulation of the cardiovascular system [20]. It involves the statistical linear or non-linear (complexity) assessment of the train of cardiac beat-to-beat interval cycles. Interpretation requires apriori knowledge of the physiological background of the heart beat sequences such as sympathetically and vagally mediated rhythms. In previous studies linear fHRV analyses, both in the time and frequency domains, were proven viable in the fetus $[21,22,24]$. The variant linear HRV parameters serve as markers for the two branches of the autonomic nervous system [1]. Those linear parameters indicating overall $\mathrm{fHRV}$ reflect the influence of both branches of the autonomic nervous system, while short-term fHRV parameters have been suggested as emphasizing vagal influences [20]. Hence the ratio between overall and short-term fHRV has been described as representing a measure of sympatho-vagal balance [3, 24]. While the classical parameters of the time domain such as the standard deviation of the normal to normal beat intervals (SDNN) and the root mean square of successive differences of the normal beats (RMSSD) have been commonly used in earlier studies on fHRV, the representation of the frequency spectrum in the human fetus is currently a matter of debate [3, 8, 22]. In addition, several measures which focus on the assessment of complex autonomic communication have been developed and applied to fHR time series [6, 21].

From previous investigations it can be expected that fHRV parameters provide an excellent tool to determine the state of the autonomic fHR regulation throughout the gestational changes and the variant states of fetal activ- ity. In this investigation discussion on the frequency spectrum of the human fetus is avoided, the focus will be on the linear fHRV of the time domain and non-linear analysis of the fetal heart rate pattern.

The aim of the present study is to describe developmental aspects of the fetal autonomic nervous system by relating numerics to visual descriptions of fetal heart rate patterns throughout the third trimester of pregnancy.

\section{Materials and methods}

\section{Subjects}

We retrieved a sample of singletons from pregnancies of normal course and perinatal outcome from our database of more than 300 fetal magnetocardiography recordings. The subjects were all studied on a singular occasion between $24+1$ and $41+6$ weeks of GA. Recordings with a duration of $<5 \mathrm{~min}$ and those technically of low quality as regarding the fHRV from the train of fetal QRS complexes (see below) were excluded from the study sample. Small for GA fetuses detected by ultrasound were excluded, as were women receiving medication known to have cardiac side effects. All subjects were informed about the fMCG study in advance and written consent was obtained prior to investigation. The study was approved by the Ethics Committee at the Medical Faculty of the Friedrich-Schiller University of Jena, Germany. The final sample consisted of 103 fetuses whose data underwent statistical analyses.

\section{Data acquisition}

All measurements were taken in the magnetically shielded room (AK 3b, Vakuumschmelze Hanau, Germany) of the Biomagnetic Centre, Friedrich Schiller University Jena. The facilities provide a 31 channel SQUID gradiometer system (Philips Biomagnetometer, Hamburg, Germany) consisting of first order gradiometers (coil diameter $20 \mathrm{~mm}$, baseline $70 \mathrm{~mm}$, diameter of the array $145 \mathrm{~mm}$ ). The pregnant women were positioned supine or with a slight twist to either side to prevent compression of the inferior vena cava by the pregnant uterus. The Dewar was positioned with its curvature above the fetal heart after sonographic localization as close to the maternal abdominal wall without contact as possible.

The SQUID signal was recorded over a period of five minutes with a sampling rate of $1000 \mathrm{~Hz}$ using a filtering band-pass between 0.3 and $500 \mathrm{~Hz}$ (CURRY, Neuroscan, Neurosoft, Sterling, VA, USA). Simultaneously, a single lead ECG of the mother was recorded to distinguish between maternal and fetal cardiac activity.

The initial raw data underwent smoothing by a polynomial filter to increase the performance of an algorithm described in detail by Schneider et al. [19]. Detection of the heartbeats was based on maximum coherence matching (MCM) to a representative QRS complex in the smoothed data. MCM was first performed in the maternal ECG to average and remove the maternal cardiac excitation cycles in each of the magnetic channels of the raw data set. MCM of the fetal signals was, consecutively, performed in the magnetic channel with the highest signal-to-noise ratio to obtain the list of the time instants of the fetal heart beats $[7,19]$. 


\section{Visual assessment of fetal heart rate patterns}

The fetal heart rate pattern (fHRP) was visualized from the train of beat-to-beat intervals and presented in a CTG-like fashion. As the gold standard of this study, the assessment of the fHRP representation was based on the criteria given in Table 1. The criteria established by Nijhuis et al. [11] are defined from 32 weeks of gestation onwards. Pillai and James [16] found correspondingly that a stable representation of typical heart rate patterns in accordance to the other variables of the fetal state can be assumed after 35 completed weeks of gestation. Therefore it was necessary to create related objective criteria reflecting the development of earlier GA as well.

With this intention, the sample was divided into three subgroups of GA (group 1: $24+1-32+0$ weeks, group 2: $32+1-35+0$ weeks, group 3: $35+1-41+6$ weeks, Table 1). The fHRP were classified into fHRP I-III in accordance to fHRP A, B, D as described by Nijhuis et al. from 32 completed weeks onwards (groups 2 and 3). Prior to 32 weeks a "quiet" pattern was distinguished from an "active" pattern (group 1) assigned as fHRP I and fHRP II, respectively. This classification was performed by an obstetrician with daily routine experience of nonstress test assessment and blinded to the results of the fHRV analysis.

\section{Data analysis}

Segments for fetal heart rate analysis (fHRV) were standardized to 256 beats [18]. A single segment per recording was chosen by a non-medical collaborator according to the following two criteria. 1) The segment was started when visual fHRP in the recorded set could be assumed unequivocally. 2) Up to $3 \%$ of undetected single heart beats were tolerated and linearly inter- polated. Otherwise, the data set was eliminated due to bad signal-to-noise ratio [19].

\section{fHRV parameters}

The following parameters of fHRV were calculated [7, 20]:

Linear fHRV measures: $\mathrm{mHR}$ - mean fetal heart rate during the selected segment, SDNN (standard deviation of normal-tonormal beat intervals, $\mathrm{ms}$ ) - overall variability of sympathetic and vagal oscillations in the short data windows investigated here. RMSSD (root mean square of successive differences, ms) reflecting vagal control. For reasons stated before, spectral analysis of the fHR is avoided and the SDNN/RMSSD (ratio between SDNN and RMSSD) is introduced, relating overall variability to its short-term variability share in the time domain. We propose this new parameter as a possible marker to reflect sympathovagal balance as a substitution for the LF/HF ratio of the spectral power between the low $(0.04-0.15 \mathrm{~Hz})$ and high frequency $(0.15-0.4 \mathrm{~Hz})$ bands as defined by international standards $[20,24]$.

Non-linear fHRV measures - complexity: PE_Mean (mean permutation entropy over a prediction range up to $2 \mathrm{~s}$ ) - high values of PE_Mean reflect high complexity of the heart rate time series [12]. Details are given in Appendix 1.

\section{Statistical methods}

Statistics were performed using the statistical software package SPSS (SPSS for Windows 10.0.7, SPSS Inc., Chicago, USA). The discriminatory values for each of the fHRV parameters were tested non-parametrically by the Mann-Whitney-U-test between two subgroups. The correlations were analyzed by Spearman's rank correlation test (two-tailed).

Table 1 Definition criteria for fHRP I-III and the subgroups in the studied sample.

\begin{tabular}{|c|c|c|c|}
\hline $\begin{array}{l}\text { Subgroups } \\
\text { gestational age }\end{array}$ & $\begin{array}{l}\text { Heart rate pattern I } \\
\text { minimum } 3 \text { min duration }\end{array}$ & $\begin{array}{l}\text { Heart rate pattern II } \\
\text { minimum } 3 \text { min duration }\end{array}$ & $\begin{array}{l}\text { Heart rate pattern III } \\
\text { minimum } 3 \text { min duration }\end{array}$ \\
\hline $\begin{array}{l}\text { Group } 1 \\
24+1-32+0\end{array}$ & $\begin{array}{l}\text { Stable heart rate (variation of } \\
\text { visually determined floating } \\
\text { baseline }<10 \mathrm{bpm} / 3 \mathrm{~min} \text { ) with } \\
\text { small oscillation bandwidth } \\
\text { ( }< \pm 5 \mathrm{bpm} \text { from floating baseline } \\
\text { fHR), isolated accelerations } \\
\text { ( }>15 \mathrm{bpm} \text { over }>15 \mathrm{~s}) \text {, floating }\end{array}$ & $\begin{array}{l}\text { Unstable fetal heart rate } \\
\text { with variant floating } \\
\text { baseline fHR not } \\
\text { exceeding } 160 \mathrm{bpm} \text {, with } \\
\text { oscillations }> \pm 5 \mathrm{bpm} \text {, } \\
\text { accelerations (if any) may } \\
\text { exceed } 160 \mathrm{bpm}\end{array}$ & - \\
\hline
\end{tabular}

$32+1-35+0$ exceed $160 \mathrm{bpm}$

Group 3

$35+1-41+6$

\author{
Variant fetal heart rate with \\ oscillation bandwidth exceeding \\ $\pm 5 \mathrm{bpm}$ from floating baseline, \\ frequent accelerations $(>15$ \\ bpm, >15 s), fHR exceeding \\ $160 \mathrm{bpm}$ only during \\ accelerations
}

\author{
Unstable fHR with frequent or \\ long-lasting and large \\ accelerations, floating baseline \\ fHR may exceed $160 \mathrm{bpm}$, \\ oscillation bandwidth in \\ between accelerations \\ exceeding $\pm 10 \mathrm{bpm}$ from \\ floating baseline fHR \\ Heart rate pattern $D$
}

Heart rate pattern B

Reference to
Nijhuis et al., 1982

Heart rate pattern $A$

Characterization of fHRP according to Nijhuis et al. 1982 from 32 weeks GA onwards [11]: fHRP A - heart rate stable, with a small oscillation bandwidth. Isolated accelerations occur. These are strictly related to movements. fHRP B - Heart rate with a wider oscillation bandwidth than fHRP A and frequent accelerations during movements. fHRP D - Heart rate unstable, with large and longlasting accelerations, frequently fused into sustained tachycardia. 


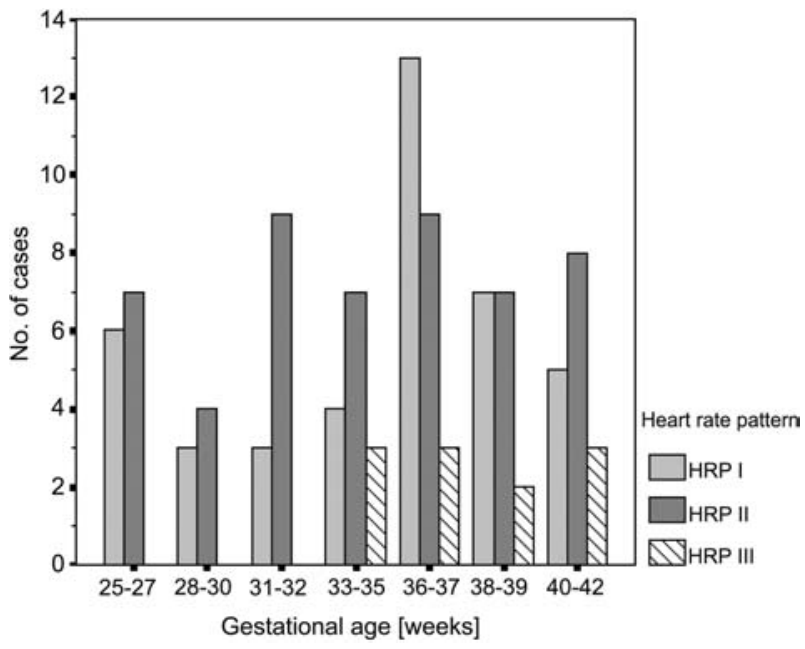

Figure 1 Distribution of the heart rate pattern assignment in the study sample. For population statistics see Table 2.

\section{Results}

The distribution of the visually classified fHRP over the total study sample is given in Figure 1, and the results of the visual classification procedure are shown in Table 2. FHRP I was observed in 41/103 cases (39.8\%) and fHRP II was observed in 51/103 cases (49.5\%). Of those fetuses beyond 32 completed weeks GA, 29/71 (40.8\%) were assumed to display fHRP I, 31/71 cases (43.7\%) fHRP II, and $11 / 71$ cases $(15.3 \%)$ fHRP III. Before 32 weeks GA $12 / 32$ fetuses (37.5\%) with quiet fHRP (I) and 20/32 cases $(62.5 \%)$ with active fHRP (II) were identified.

The individual distributions of the fHRV parameters (SDNN, RMSSD, PE_Mean, mHR) depending on GA are shown in Figure 2. The single values of all the studied parameters are distributed over a wide range. Nevertheless, there is some indication of a slight increase of SDNN and RMSSD, but a decrease of PE_Mean with GA (Figure 2). The correlations are given in Table 3. More clearly the parameters cluster according to their visual assignments across the age groups for fHRP I and II. The values representing fHRP III beyond 35 weeks GA form an additional characteristic cluster in each of the scatter plots. They are associated with higher values of $\mathrm{mHR}$ and
SDNN, intermediate values of RMSSD and low complexity (PE_Mean).

The characteristics of the distributions within the GA groups are shown in Figure 3. In each of the studied gestational periods both SDNN and RMSSD were significantly higher in fHRP II than in fHRP I (Figure 3A and B). This was associated with significantly lower levels of complexity (Figure 3C). There were no significant differences across the age groups within fHRP I and II except for SDNN between subgroups 1 and 3 of fHRP II $(P=0.04)$. The $m H R$, in contrast, was not significantly different between fHRP I and fHRP II (Figure 3D). The numerical data summarizing the distributions within the 8 subgroups are shown in Table 4, and the levels of significance are shown in Figure 3A-D.

With regard to fHRP III, SDNN was similar between fHRP II and III, but RMSSD was significantly lower in fHRP III beyond 35 weeks GA (Figure $3 \mathrm{~A}$ and B). The same was strongly pronounced for PE_Mean (Figure $3 C$ ). There was also a significant difference in the two GA subgroups $(P=0.01$; Mann-Whitney $U)$. As expected, these results were accompanied by higher $\mathrm{mHR}$ in fHRP III near term (>35 weeks GA, Figure 3D).

The dependencies of the SDNN/RMSSD ratio are shown in Figure 4. The significant negative correlation between SDNN/RMSSD and PE_Mean was essentially based on the fHRP clustering from fHRP I with lowest SDNN/RMSSD and highest PE_Mean over fHRPII to fHRP III with highest SDNN/RMSSD and lowest PE_Mean (Figure 4A). The SDNN/RMSSD ratio did not change with GA for fHRP II $(r=0.015, P=0.92)$. In contrast, fHRP I was associated with a decreasing SDNN/ RMSSD ratio with advancing gestation (Figure 4B, $r=0.32, P=0.042)$.

Near term we observed a significant increase in the SDNN/RMSSD ratio with increasing fetal activity (Figure 4C). There were no significant differences between fHRP I and fHRP || in earlier gestational weeks (data not shown).

\section{Discussion}

In our study we utilized beat-to-beat heart rate variability (fHRV) retrieved from non-invasive magnetocardiographic

Table 2 Distribution of the study sample between the GA groups and fHRP assignments.

\begin{tabular}{lllll}
\hline Subgroups & Fetal HRP I & Fetal HRP II & Fetal HRP III & Overall \\
\hline Group 1 & 12 & 20 & - & 32 \\
Group 2 & {$[29.3 \%],(37.5 \%)$} & {$[39.2 \%],(62.5 \%)$} & & {$[31.1 \%],(100 \%)$} \\
& 4 & 7 & 3 & 14 \\
Group 3 & {$[9.8 \%],(28.6 \%)$} & {$[13.7 \%],(50 \%)$} & {$[27.3 \%],(22.4 \%)$} & {$[13.6 \%],(100 \%)$} \\
\multirow{2}{*}{ Overall } & 25 & 24 & 8 & 57 \\
& {$[60.9 \%],(43.9 \%)$} & {$[47.1 \%],(42.1 \%)$} & {$[72.7 \%],(14.0 \%)$} & {$[55.3 \%],(100 \%)$} \\
& 41 & 51 & 11 & 103 \\
\end{tabular}



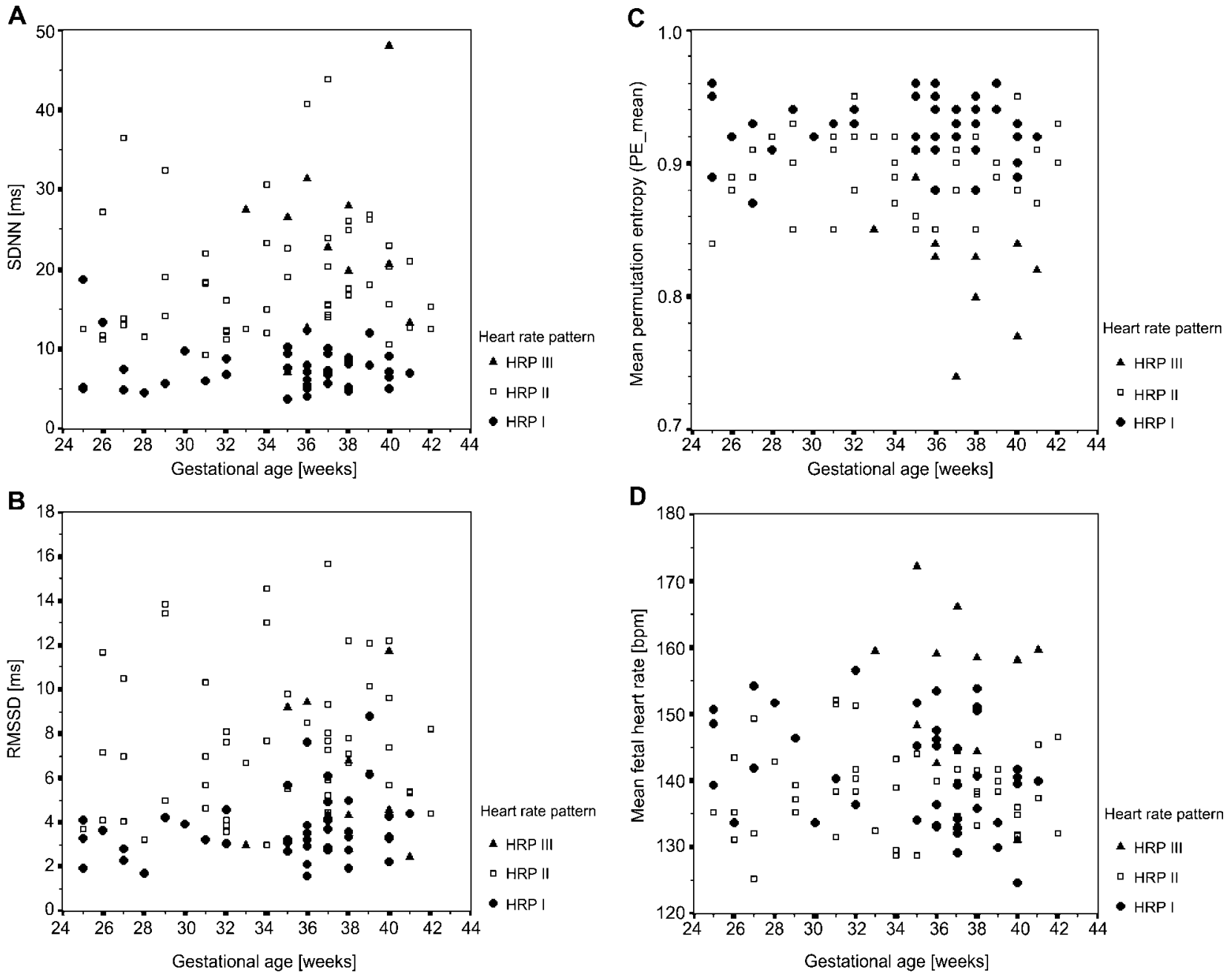

Figure 2 Scatter plots of the distribution of the studied fHRV parameters over the studied period of gestation in relationship to the fHRP assignment A) SDNN, B) RMSSD, C) PE_Mean, D) mean fHR (abbreviations: see text). Numerical data is summarized in Table 3.

recordings as a measure to describe developmental aspects of the sympatho-vagal heart rate regulation in the human fetus. For the first time, the parameters of fHRV were studied in their gestational course and related to visual information representing different levels of fetal activity.
We were able to show that the typical heart rate patterns are distinctively described by the application of fHRV from about 25 weeks GA onwards. The pre-classification of fHRP led to a plausible and characteristic clustering of $\mathrm{fHRV}$ parameters across the gestational period studied here.

Table 3 Correlations between the studied fHRV parameters and GA.

\begin{tabular}{lccrr}
\hline Correlation & GA & & & \\
\cline { 2 - 5 } & Overall & fHRPI & fHRPII & fHRPIII \\
\hline SDNN & 0.08 & -0.13 & 0.12 & 0.04 \\
RMSSD & 0.08 & 0.253 & 0.10 & 0.12 \\
PE_Mean & -0.06 & 0.03 & 0.19 & -0.66 \\
mHR & -0.03 & -0.29 & -0.02 & -0.24 \\
SDNN/RMSSD & -0.02 & -0.32 & -0.02 & 0.34 \\
\hline
\end{tabular}

Levels of significance: $\mathrm{P}<0.05$, PE_Mean vs. HRP III correlation -0.66 ; $\mathrm{P}<0.05$, SDNNN/RMSSD vs. HRP I correlation $-0.32 ; \mathrm{P}<0.1$, mHR vs. HRP I correlation -0.29 . 
A

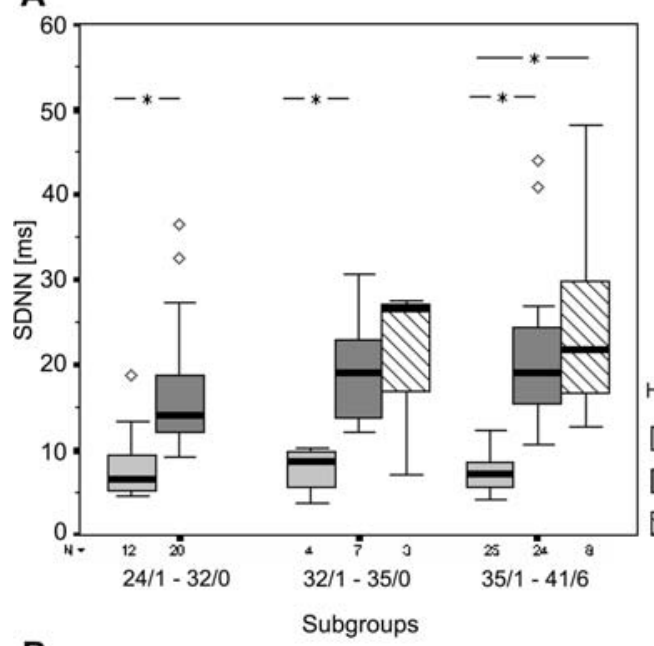

B

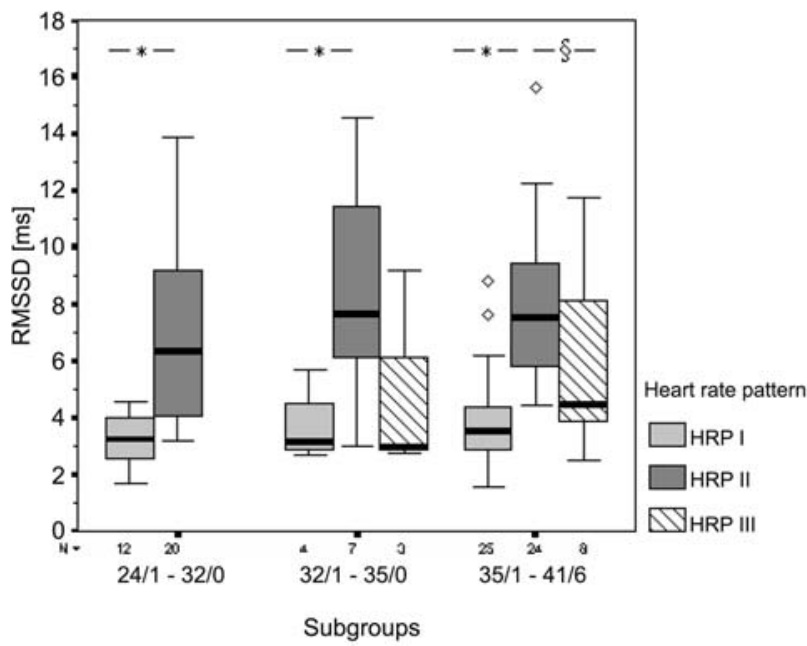

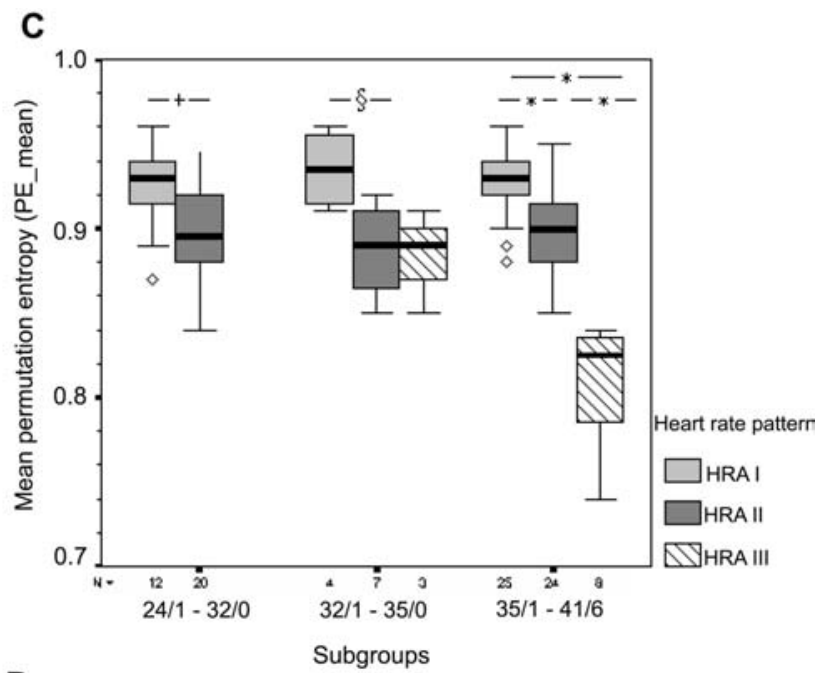

D

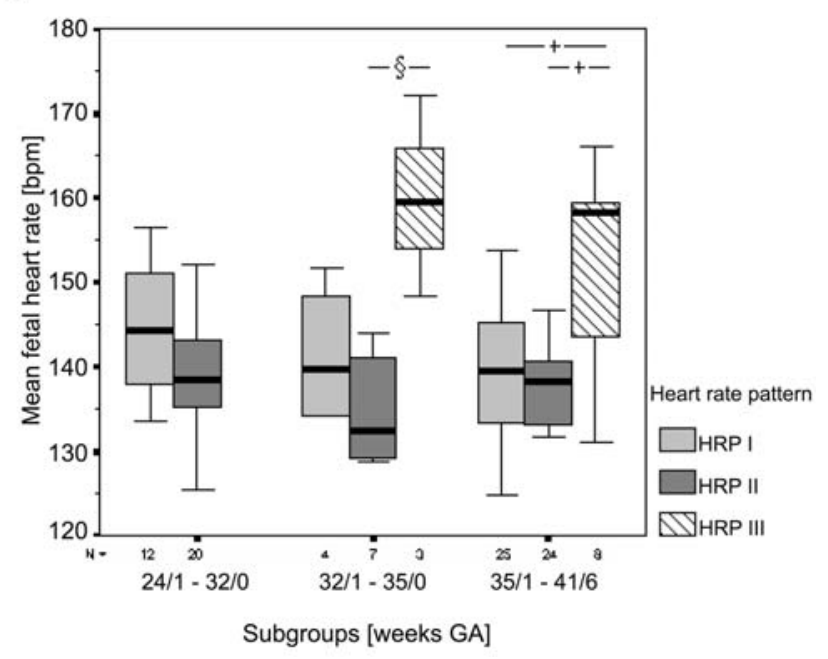

Figure 3 Box plots of the distribution of the studied parameters of fHRV in relationship for the defined subgroups according to the level of maturation and the fHRP assignment. A) SDNN, B) RMSSD, C) PE_Mean, D) mean fHR (abbreviations: see text, levels of significance: $\left.{ }^{*} \mathrm{P}<0.001 ;{ }^{+} \mathrm{P}<0.01 ;{ }^{\circledR} \mathrm{P}<0.05\right)$.

FHRP assumed to represent low level fetal activity were accompanied by low range linear fHRV parameters and associated with the highest levels of complexity in the time series. The significantly decreasing ratio of SDNN/RMSSD with GA in this subset indicates an increasing level of vagal influence towards term as has been previously proposed [12, 24].

The fHRP II beyond 32 weeks GA represent the state $2 \mathrm{~F}$ as described by Nijhuis et al. As could be expected, $\mathrm{mHR}$ was not significantly different in these more active

Table 4 Distribution characteristics (median; $25^{\text {th }} ; 7^{\text {th }}$ percentile) of the studied fHRV parameters in the subgroups.

\begin{tabular}{llcllll}
\hline Gp. & fHRP & SDNN [ms] & RMSSD [ms] & mHR [bpm] & PE_Mean & SDNN/RMSSD \\
\hline 1 & I & $6.5(5.0 ; 9.6)$ & $3.3(2.4 ; 4.0)$ & $144.2(137.2 ; 151.4)$ & $0.930(0.913 ; 0.940)$ & $2.52(1.65 ; 3.19)$ \\
& II & $14.0(11.9 ; 18.8)$ & $6.3(4.1 ; 9.8)$ & $138.4(135.2 ; 143.4)$ & $0.895(0.880 ; 0.920)$ & $2.80(1.99 ; 3.21)$ \\
2 & I & $8.6(5.7 ; 9.9)$ & $3.2(2.9 ; 4.5)$ & $139.6(134.1 ; 148.4)$ & $0.935(0.915 ; 0.955)$ & $2.07(1.60 ; 2.72)$ \\
& II & $19.1(13.8 ; 23.0)$ & $7.7(6.1 ; 11.4)$ & $132.5(129.1 ; 141.1)$ & $0.890(0.865 ; 0.910)$ & $2.10(1.84 ; 2.89)$ \\
& III & $26.5(16.8 ; 27.0)$ & $3.0(2.9 ; 6.1)$ & $159.6(154.0 ; 165.8)$ & $0.890(0.870 ; 0.900)$ & $2.89(2.74 ; 6.02)$ \\
3 & I & $7.2(5.7 ; 8.6)$ & $3.5(2.9 ; 4.4)$ & $139.5(133.4 ; 145.3)$ & $0.930(0.920 ; 0.940)$ & $1.95(1.60 ; 2.53)$ \\
& II & $19.2(15.4 ; 24.5)$ & $7.5(5.8 ; 9.5)$ & $138.2(133.2 ; 140.7)$ & $0.900(0.880 ; 0.915)$ & $2.64(2.15 ; 3.22)$ \\
& III & $21.8(16.6 ; 29.7)$ & $4.5(3.9 ; 8.1)$ & $158.3(143.6 ; 159.4)$ & $0.825(0.785 ; 0.835)$ & $4.33(3.94 ; 4.92)$ \\
\hline
\end{tabular}



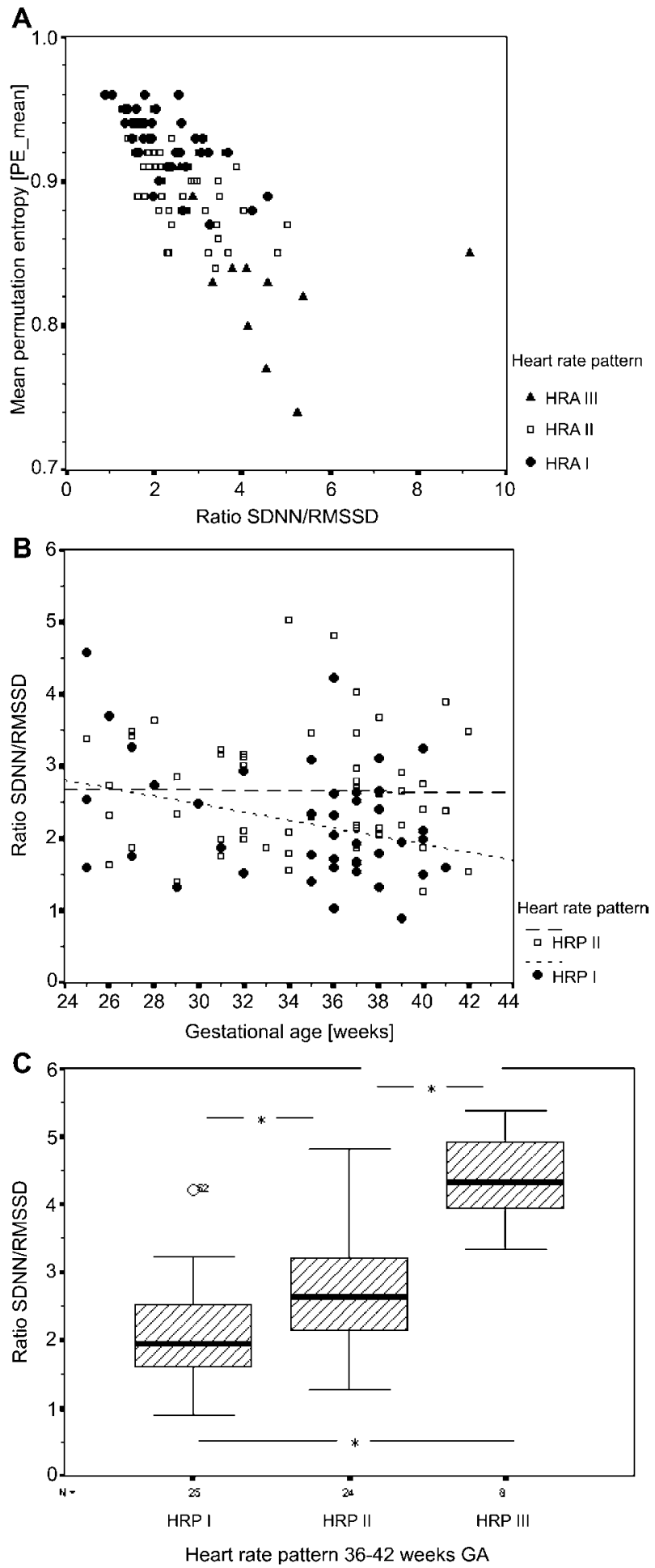

Figure 4 SDNN/RMSSD ratio and complexity.

A) High SDNN to the cost of low RMSSD is associated with low complexity (low PE_Mean). B) Development of SDNN/RMSSD with gestational age in the studied sample. Only fHRP I and II are considered. Note the significant decrease $(P=0.042)$ in SDNN/RMSSD with GA only in fHRP I cases (abbreviations: see text). C) Increase of SDNN/RMSSD with increasing fetal activity levels beyond 35 weeks $\mathrm{GA}\left({ }^{*} \mathrm{P}<0.001\right)$. patterns than those representing fetal quiescence [23]. We did not see a difference between those cases studied prior to and beyond 32 weeks GA in the relationships between fHRP I and II. FHRP II was associated with significantly higher SDNN and RMSSD and lower complexity levels. However, the ratio of SDNN/RMSSD only became significantly different after 35 weeks GA. This finding indicates that the previously described stabilization of the variables at this GA appears to be accompanied by a stabilization of sympatho-vagal balance [16, 22, 24].

The fHRP assigned to type III patterns were characterized by a significantly higher mHR. This may have led to the classification of those three cases between 32 and 35 weeks of gestation. Interestingly, while near term (>35 weeks GA) the fHRP III were characterized by a significantly different way of clustering within all three studied parameters of fHRV, prior to that age the assigned cases did not show the characteristic low complexity features as can be observed from Figures $2 \mathrm{C}$ and 3C.

Van Leeuwen et al. investigated both linear parameters in the time domain and complexity measures, namely the approximate entropy, in fMCG data [21]. They demonstrated an overall increase in the parameters of the time domain as an expression of increasing fHRV with advancing GA accompanied by diverse results towards term. The level of fetal activity in addition to advancing gestation is the likely influence to explain these data. We could reproduce the increase in the fHRV parameters of the time domain with GA though not on a significant level. These increasing values of SDNN and RMSSD reflect the increasing amplitudes of the respective heart rate fluctuations during maturation. The decreasing values of the PE_Mean are related to decreasing complexity of the heart rate time series that is accompanied by higher levels of fetal activity later in gestation. These variant results may have been influenced by methodological differences. The length of the time series has a significant impact on the results [18]. To reduce the likelihood of covering state changes, we standardized our segments of interest to 256 beats. Further investigations are required to differentiate between methodological and physiological effects.

The LF/HF ratio has up to now been regarded as an expression of the sympatho-vagal balance of the fetus [24]. The same authors describe a decrease of LF/HF with advancing gestation as suggesting an increased vagal influence on fHR regulation. Van Leeuwen et al. were able to observe that the development in the spectral power bands passes a critical period between 28-31 weeks GA, leaving the LF/HF ratio almost uninfluenced [22]. David et al. [3] proposed boundaries of the HF, LF, and VLF bands differing from the standards in order to express the development of sympatho-vagal balance in the last trimester. They found a decreasing 
VLF/HF ratio, but an unchanged LF/HF ratio. Taking into account the general correlations between RMSSD and $\mathrm{HF}$ as well as between SDNN and VLF, these findings support the approach in the time domain presented here $[3,20]$. For reasons stated before, we did not apply parameters of the frequency domain in our current study. Instead we introduced a new parameter, the SDNN/ RMSSD ratio, as an expedient to estimate the share of short-term in relation to overall variability as an expression of sympatho-vagal balance in the time domain. The previously described decrease in the LF/HF ratio with gestational age could be reproduced in the SDNN/ RMSSD ratio [12, 22, 24].

Mean FHR was found to be a confounding variable to fHRV analysis. In their study, Lange et al. [9] found that a low mean RR interval, corresponding to higher mHR, could be shown to correlate to lower fHRV parameters and the share of recordings with larger mean RR intervals was observed beyond 30 weeks of gestation. In addition, it should be important to note that $\mathrm{mHR}$ must not be mistaken for the baseline FHR. Furthermore, mHR cannot be interpreted independently from visual state classification especially in late gestation. While basal FHR decreases with advancing gestation, increased $\mathrm{mHR}$ is a major characteristic of fetal active waking $[4,11,15,16]$. Interestingly, despite highest levels of mHR in fHRP III, we were able to observe the highest SDNN in our sample here.

The pre-classification of fetal activity levels from a visual inspection of fHR patterns is not the current multimodal state-of-the-art method to assign the fetal behavioral state $[4,11]$. Ultrasound observation during fMCG recordings is not applicable because of its magnetic interference on the signal. Doppler-beam based methods of fHR analysis constitute the clinical standard for 'intermediate' - and long-term fetal surveillance [10, 14]. Such results may be used to calculate the parameter set referred to as the Dawes-Redman-criteria, which include short-term variability (STV) calculations from average interbeat-intervals of about 7-11 fetal heartbeats per sample [13]. Given the strong association between HRV measures and brain stem activity, our data suggest that the fetal heart rate pattern alone may be a much more valuable parameter for the level of autonomic nervous control than the temporal coincidence of the classical fetal state variables, especially prior to 32 weeks' gestation. Further investigation into longer time series and the relation between the time domain parameters and the recently proposed specific fetal frequency bands will be necessary to prove this, and may potentially benefit prenatal diagnostics.

fHRV and complexity indices mirror essential developmental aspects of the autonomic nervous system with an accuracy which can fundamentally not be obtained by the established CTG technology. We were able to document a clear developmental progress leading to signif- icantly distinct levels of sympathetic and vagal influence on fHR as gestation progresses, and to the appearance of high activity level representation in the fHRP accompanied by the lowest levels of complexity in the time series of the fetal heart beats. We conclude that a combined analysis of fHRV, based on SDNN/RMSSD and PE_Mean, and fHRP is advantageous in the assessment of maturation of the fetal autonomic nervous system.

\section{Appendix 1}

\section{Complexity estimation}

The complexity was estimated based on permutation entropy (PE). PE is a complexity measure for time series operating on an ordinal level [2]. Its main advantages are robustness with respect to noise, its applicability to short time series and its easy estimation. PE measures the entropy of sequences of ordinal patterns derived from $\mathrm{m}$-dimensional delay embedding vectors. In the following we briefly summarize the definition of the permutation entropy. More details can be found in [2].

The scalar time series $\{x(t)\}_{t=1}^{T}$ is embedded into an $\mathrm{m}$-dimensional space

$X_{t}=[x(t), x(t+L), \ldots, x(t+(m-1) L]$

where $m$ is called the embedding dimension and $L$ the embedding delay time. In general, there are $m$ ! possible order patterns, which is the number of permutations of the $m$ coordinates in $X_{t}$. Now, let $p(\pi)$ denote the relative frequency of order pattern $\pi$ :

$p(\pi)=\frac{\#\left\{t \mid 1 \leq t \leq T-(m-1) L, \text { where } X_{t} \text { has type } \pi\right\}}{T-(m-1) L}$

Then, for fixed embedding dimensions $m \geq 2$, and fixed delay $L$, permutation entropy is defined as

$P E(m ; L)=-\sum_{\pi} p(\pi) \log _{2} p(\pi) / \log _{2} m !$,

where the sum runs over all $m$ ! patterns $\pi$.

In our calculations the embedding dimension 3 is used because of the short length of data sequences. PE_Mean was calculated as mean of the PE values of embedding delay times $L$ between 0.1 and $2 \mathrm{~s}$.

\section{References}

[1] Akselrod S, Gordon D, Ubel FA, Shannon DC, Berger AC, Cohen RJ. Power spectrum analysis of heart rate fluctuations: a quantitative probe of beat-to-beat cardiovascular control. Science. 1981;213:220-2. 
[2] Bandt C, Pompe B. Permutation entropy: a natural complexity measure for time series. Phys Rev Lett. 2002;88: 174102.

[3] David M, Hirsch M, Karin J, Toledo E, Akselrod S. An estimate of fetal autonomic state by time-frequency analysis of fetal heart rate variability. J Appl Physiol. 2007;102: 1057-64.

[4] Di Pietro JA, Hodgson DM, Costigan KA, Hilton SC, Johnson TRB. Fetal neurobehavioural development. Child Development. 1996;67:2553-67.

[5] Di Pietro JA, Costigan KA, Pressmann EK. Fetal state concordance regulation predicts infant state regulation. Early Hum Dev. 2002;68:1-13.

[6] Frank B, Pompe B, Schneider U, Hoyer D. Permutation entropy improves fetal behavioural state classification based on heart rate analysis from biomagnetic recordings in near term fetuses. Med Biol Eng Comp. 2006;44:17987.

[7] Grimm B, Haueisen J, Huotilainen M, Lange S, Van Leeuwen P, Menendez T, et al. Recommended standards for fetal magnetocardiography. Pacing Clin Electrophysiol. 2003;26:2121-6.

[8] Hartmann M, van Leeuwen P, Gronemeyer D. [Fetal heart rate variability in pregnancy. Spectral analysis based on magnetocardiography.] Biomed Eng. 1997;42(Suppl): 61-2.

[9] Lange S, van Leeuwen P, Geue D, Hatzmann W, Groenemeyer $\mathrm{D}$. Influence of gestational age, heart rate, gender and time of day on fetal heart rate variability. Med Biol Eng Comp. 2005;43:481-6.

[10] Miller DA, Rabello YA, Paul RH. The modified biophysical profile: antepartum testing in the 1990s. Am J Obstet Gynecol. 1996;174:812-7.

[11] Nijhuis JG, Prechtl HFR, Martin CB, Bots RSG. Are there behavioral states in the human fetus? Early Hum Dev. 1982;6:177-95.

[12] Ohta T, Okamura K, Kimura Y, Suzuki T, Watanabe T, Yasui $\mathrm{T}$, et al. Alteration in the low-frequency domain in power spectral analysis of fetalheart beat fluctuations. Fetal Diagn Ther. 1999;14:92-7.

[13] Pardey J, Moulden M, Redman WD. A computer system for the numerical analysis of non-stress tests. Am J Obstet Gynecol. 2002;186:1095-103.
[14] Peters M, Crowe J, Pieri JF, Quartero H, Hayes-Gill B, James $\mathrm{D}$, et al. Monitoring the fetal heart non-invasively: a review of methods. J Perinat Med. 2001;29:408-16.

[15] Pillai M, James D. The development of fetal heart rate patterns during normal pregnancy. Obstet Gynecol. 1990; 76:812-6.

[16] Pillai M, James D. Behavioural states in the normal mature human fetus. Arch Child Disease. 1990;65(1 Spec No): 39-43.

[17] Pillai M, James D. Development of human fetal behavior: a review. Fetal Diagn Ther. 1990;5:15-32.

[18] Ronneburger A, Kähler C, Hoyer D, Schleussner E, Schneider $\mathrm{U}$, Haueisen $\mathrm{J}$, et al. Influence of fetal state of activity and duration of recording time on linear and non-linear parameters of fetal heart rate variability investigated by fetal magnetocardiography. Biomed Eng. 2004;48(Suppl): 143.

[19] Schneider U, Schleussner E, Haueisen J, Nowak H, Seewald HJ. Signal analysis of auditory evoked cortical fields in fetal magnetoencephalography. Brain Topogr. 2001;14: 69-80.

[20] Task Force. Heart rate variability. Standards of measurement, physiological interpretation, and clinical use. Task force of the European society of cardiology and the north American society of pacing and electrophysiology. Eur Heart J. 1996;17:354-81.

[21] Van Leeuwen P, Lange S, Bettermann H, Groenemeyer D, Hatzmann W. Fetal heart rate variability and complexity in the course of pregnancy. Early Hum Dev. 1999;54:259-69.

[22] Van Leeuwen P, Geue D, Lange S, Hatzmann W, Groenemeyer D. Changes in the frequency power spectrum of fetal heart rate in the course of pregnancy. Prenat Diagn. 2003;23:909-16.

[23] Visser GH, Mulder EJ, Stevens H, Verweij R. Heart rate variation during fetal behavioural states 1 and 2. Early Hum Dev. 1993;34:21-8.

[24] Zhuravlev Y, Rassi D, Mishoin AA, Emery SJ. Dynamic analysis of beat-to-beat fetal heart rate variability recorded by SQUID magnetometer: quantification of sympathovagal balance. Early Hum Dev. 2002;66:1-10.

Received December 21, 2007. Revised March 22, 2008. Accepted April 15, 2008. Previously published online June 12, 2008. 